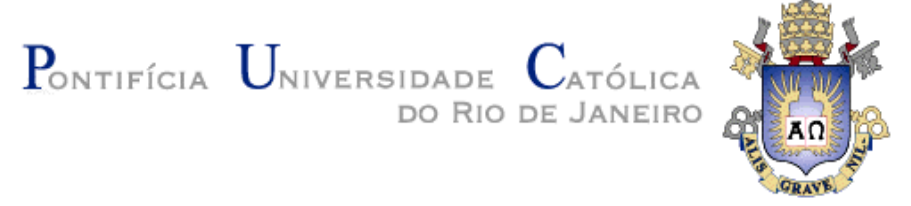

Flávia Monteiro de Castro Souza

JUVENTUDE E MOVIMENTO HIP-HOP:

a construção de identidade, luta por direitos

e cidadania

Dissertação de Mestrado

Dissertação apresentada ao Programa de PósGraduação em Serviço Social da PUC-Rio como requisito parcial para obtenção do título de Mestre em Serviço Social.

Orientadora: Prof. Andréia Clapp Salvador 
Flávia Monteiro de Castro Souza

\section{JUVENTUDE E MOVIMENTO HIP-HOP: a construção de identidade, luta por direitos e cidadania}

Dissertação apresentada como requisito parcial para obtenção do grau de Mestre pelo Programa de Pós-Graduação em Serviço Social do Departamento de Serviço Social do Centro de Ciências Sociais da PUC-Rio. Aprovada pela Comissão Examinadora abaixo assinada.

Prof. Andréia Clapp Salvador

Orientadora

Departamento de Serviço Social PUC-Rio

Prof. Adriana Facina

Universidade Federal Fluminense

Prof. Adair Leonardo Rocha Departamento de Comunicação PUC-Rio

Prof. Denise Pini Rosalem da Fonseca Departamento de Serviço Social PUC-Rio

Prof. Mônica Herz

Vice-Decana de Pós-Graduação do Centro de Ciências Sociais - PUC-Rio

Rio de Janeiro, 08 de abril de 2010 
Todos os direitos reservados. É proibida a reprodução total ou parcial do trabalho sem autorização da universidade, do autor e do orientador.

\section{Flávia Monteiro de Castro Souza}

Graduou-se em Estudos Internacionais com especialização em Antropologia pela University of Oregon 2004, EUA. É dançarina e professora de Lindy Hop, dança negra originada nos anos 1920-40. Ensinou dança e arte na Organização Filantrópica Solar Meninos de Luz. No momento tem dedicado seus estudos às seguintes áreas: movimentos sociais culturais; cultura popular $e$ comunitária; expressões culturais e artísticas na diáspora áfrica: blues, jazz, soul e hip-hop.

Ficha Catalográfica

Souza, Flávia Monteiro de Castro

Juventude e movimento HIP-HOP : a construção de identidade, luta por direitos e cidadania / Flávia Monteiro de Castro Souza ; orientadora: Andréia Clapp Salvador. -2010.

$133 \mathrm{f.} ; 30 \mathrm{~cm}$

Dissertação (Mestrado) - Pontifícia Universidade Católica do Rio de Janeiro, Departamento de Serviço Social, 2010 .

Inclui bibliografia.

1. Serviço social - Teses. 2. Movimentos sociais culturais. 3. Hip-hop. 4. Juventude. 5. Identidade. 6. Cidadania. I. Salvador, Andréia Clapp. II. Pontifícia Universidade Católica do Rio de Janeiro. Departamento de Serviço Social. III. Título. 
Para meus pais, Dindo e Cândida, que me ensinaram muitas coisas, cada qual a sua maneira. 


\section{Agradecimentos}

Uma dissertação é fruto de um percurso significativo, onde o autor caminha com suas fontes e dialoga com todos aqueles, que estiveram presentes. Embora este seja um trabalho individual, ele nasceu de idéias, sentimentos, atos, palavras, canções, movimentos, desafios, lutas, experiências, percepções e conquistas compartilhadas nesta jornada. Dele participaram diversas pessoas que trilharam caminhos, muitas vezes imprevisíveis, mas que se cruzaram neste percurso.

Em especial, agradeço:

À minha orientadora, Andréia Clapp Salvador, pelo interesse, sensibilidade, críticas e sugestões, que criaram um espaço de acolhimento para desenvolver estas idéias. Obrigada companheira pela determinação, senso de humor e seriedade ao mergulhar comigo no mundo dos movimentos culturais e da luta através da arte.

À Denise Fonseca, primeira orientadora desta dissertação, que me apresentou alguns autores que ajudariam a germinar este estudo. Obrigada pelas críticas, sugestões e sensibilidade.

À Ana Maria Quiroga, um exemplo de luta e determinação na construção do conhecimento. Ana, obrigada por seu espírito incansável, suas aulas e sugestões.

À professora Ilda Lopes Rodrigues da Silva, pela visão de mundo tão humana e abrangente, que me ajudou a descobrir novos olhares.

A todos os professores da PUC que me influenciaram nesta jornada.

Thanks to Jeff Harrison, my very favorite teacher, who first instigated me to understand the struggles for justice and the walk towards the construction of one's identity, challenging my ideals and my world.

Ao Gas-PA do LUTARMADA, pelos depoimentos, canções, palestras, convites, e protestos que tanto contribuíram neste trabalho. Obrigada pelo compromisso e coragem de resistir a este sistema, revelando que uma nova perspectiva é possível.

Ao Luck e ao Fábio do GBCR, pela alegria de viver aparente em sua arte, pelas contribuições, convites, contatos, depoimentos e por serem exemplos de conduta e de vida para tantas pessoas.

À Queen do Rap de Saia, por ter proporcionado um espaço de discussão sobre as mulheres negras de periferia na sociedade e no hip-hop. Aprendi muito com sua dedicação, determinação e visão de mundo tão "universalista".

Ao Gouki, por todas as dicas sobre a cultura hip-hop, indicações de vídeos e leituras. E pelo tempo dedicado a cultura.

Aos funcionários do Departamento de Serviço Social: Joana pela organização e clareza. Bruno e Mariana por sempre estarem prontos. 
À Geovana, por ser luz. Pela amizade e por haver compartilhado tantas coisa.

À Adriana Severo, pelo exemplo de mulher sensível e forte.

À Mariana pela alegria de viver e a coragem de arriscar. Obrigada pela amizade.

À Jussara, pela amizade, ajuda e palavras tão sensíveis e precisas.

Ao Márcio, por me dar um voto de confiança e compartilhar em meus sonhos.

À Carla, por uma amizade que permitiu identificar nossas origens, Muriaé.

Ao Toni, pelo senso crítico que sempre instigou preciosos debates e diálogos.

À todas as colegas que contribuíram dentro e fora da sala de aula.

Ao Departamento de Serviço Social e à PUC que permitiram este percurso.

Aos meninos do RDC, que estiveram de prontidão para auxiliar se necessário.

Aos amigos do Bar da Freira, que prepararam minha comida tantas vezes.

Aos meus familiares, sempre ao meu lado e capazes de me entender mesmo quando minha atitude não fazia sentido. Obrigada pela dedicação e amor de vocês.

Aos meus avôs, Antônio Flávio e Nilza, pelo amor, pelo carinho, pela compreensão, pela roça e pela goiabada.

Ao meu pai, que mesmo com todas as turbulências de sua alma, foi capaz de me mostrar o que significa ser um coração bondoso. Muitas saudades.

À minha mãe, pela determinação, honestidade, confiança, alegria e força de vontade: qualidades que ela me ensinou com muito amor, e, mesmo em meio a diferenças, me incentivou a correr atrás de meus sonhos.

Ao meu padrasto Silvio, que esteve presente durante estes anos, escutando, compartilhando e agregando amor à família.

À Larissa, por haver compartilhado comigo os momentos mais profundos e por mostrar através de suas ações que vale a pena lutar pelos seus sonhos.

À Laura, pela esperteza e doçura, misturas em uma alma que tem muito amor e alegrias para distribuir.

À Fernanda, pela sabedoria e alegria visíveis em sua fala, seu olhar e seu jeito menino de ser.

Ao Pablo, que "chegou como quem chega do nada. E antes que eu dissesse não se instalou feito um posseiro dentro do meu coração". Obrigada pelas conversas sobre esta pesquisa; pela força, paciência, confiança, carinho, dedicação e amor.

Aos meus amigos da vida, que de várias formas me deram apoio, e sobre os quais eu deveria escrever muito mais: Cíntia, Roberta, Cláudia, Gabrielas, Roberto, Luiz, Victor, Ulla, Soneto, Nanu, Barbara, Denise, Anna, Isaac e alguns outros.

A meus amigos, dançarinos e amantes do lindy hop, que me inspiraram por meio de músicas, movimentos, olhares, sorrisos e relações construídas na dança.

Ao Frankie, pela inspiração e todos os swing outs.

A Deus, por me dar a vida. Pela coragem e a força de vontade para tentar fazer dela o melhor, lutando por meus sonhos e tudo aquilo que eu entendo como importante, valioso e verdadeiro. 


\section{Resumo}

Souza, Flávia Monteiro de Castro; Salvador, Andréia Clapp. Juventude e Movimento Hip-Hop: a construção de identidade, luta por direitos e cidadania. Rio de Janeiro, 2010. 133p. Dissertação de Mestrado Departamento de Serviço Social, Pontifícia Universidade Católica do Rio de Janeiro.

A dissertação em questão oferece uma reflexão acerca dos movimentos sociais culturais da atualidade, especificamente do Movimento Hip-Hop. Inicialmente foi realizada uma fundamentação teórica onde privilegiei algumas categorias de análise pertinentes ao tema de estudo, como movimento social cultural e identidade. Em seguida mostrei um pouco da história da construção cultural das Américas e do surgimento das expressões culturais e artísticas na diáspora africana. Foram ressaltados significativos aspectos dos Griots africanos, da escravidão, do Blues, do Jazz, do Lindy Hop, do Soul, do Funk e do Hip-Hop. Em seguida foram entrevistados líderes militantes dos seguintes grupos de hiphop da periferia do Rio de Janeiro: o Coletivo Hip-Hop LUTARMADA, o Grupo Breaking Consciente da Rocinha e o Rap de Saia. A pesquisa buscou conhecer o olhar que as lideranças destes coletivos têm com relação à arte como instrumento de transformação, à identidade, à luta por direitos e ao exercício da cidadania. Buscou-se também, demarcar a influência destas visões na vida dos integrantes das organizações. O objetivo do trabalho foi, a partir da análise do discurso destes atores, conhecer as representações sociais que estes têm sobre a cultura hip-hop carioca, enquanto expressão artística e cultural e ação política. Entre as questões mais presentes nos depoimentos estavam: o aspecto identitário incluindo raça, gênero e classe; a paixão pela cultura hip-hop; a associação do hip-hop com o conhecimento; e diferentes ideologias quanto a transformação social. $\mathrm{O}$ aspecto mais marcante do movimento cultural do hip-hop, diz respeito a pluralidade deste movimento cultural, que ressalta ideologias diversas, muitas vezes antagônicas, e que revelam desde visões revolucionárias à luta por mudança pessoal.

\section{Palavras-chave}

Movimentos Culturais; Hip-Hop; Identidade; Cidadania. 


\section{Abstract}

Souza, Flávia Monteiro de Castro; Salvador, Andréia Clapp (Advisor). Youth and Hip-Hop Movement: the construction of identity, struggle for rights and citizenship. Rio de Janeiro, 2010. 133p. MSc. Dissertation Departamento de Serviço Social, Pontifícia Universidade Católica do Rio de Janeiro.

This thesis offers a reflection on the cultural social movements of today, more specifically on the Hip-Hop Movement. To begin this research, took place a study emphasizing a few theoretical categories essential for the process, such as cultural and social movement and identity. Then, I presented some aspects that fundament the art and culture historical journey of the Americas, highlighting its importance on the cultural and artistic expressions in the African Diaspora. The study drew attention to significant aspects of the African Griots, slavery, Blues, Jazz, Lindy Hop, Soul Music, Funk and Hip-Hop. Further on, I interviewed leaders of three hip-hop groups from the suburbs of Rio de Janeiro: Coletivo HipHop LUTARMADA, Grupo Breaking Consciente da Rocinha and Rap de Saia. The research aimed at getting to know these leaders viewpoints in relation to art as an instrument of change, to identity, to fight for rights and to citizenship. I also intended to point out the influence of the worldviews encountered in the lives of the organization members. By analyzing the discourse of these social actors, the goal of this work was to understand the social representations that these leaders have on hip-hop culture, as a form of artistic and cultural expression and of political action. Among the themes we evidenced in theirs speeches were: the identitary aspect, including race, gender and class; the passion for hip-hop culture and art; the belief that hip-hop generates knowledge; and the different ideologies referring to fight for rights and social change. The most significant aspect present in the Hip-hop cultural movement has to do with the plurality of this movement, which emphasizes different ideologies, in many cases contradictory, ranging from a revolutionary perspective to a search for personal change.

\section{Keywords}

Cultural Movements; Hip-Hop; Identity; Citizenship. 


\section{Sumário}

$\begin{array}{ll}\text { 1. Introdução } & 11\end{array}$

2. Dilemas dos movimentos sociais na contemporaneidade em um mundo neoliberal

2.1. Transformações ocorridas no Século XX: novos retratos das sociedades contemporâneas

2.2. Caminhos percorridos pelos de baixo: tomada da palavra através da cultura e política

2.3. Construção de Identidade: uma "renegociação" de poder

2.4. Movimentos Sociais, Cidadania e Luta por Direitos

3. História e Memória do Hip-Hop

3.1. Do Blues ao Jazz

3.2. Do Soul ao Funk

3.3. O Hip-Hop está aqui

3.4. Os quatro/cinco elementos do Hip-Hop

4. Vozes da Juventude de Periferia: Movimento Cultural do Hip-Hop no Rio de Janeiro

4.1. A história dos Movimentos do Hip-Hop: LUTARMADA, GBCR e Rap de Saia

4.2. Propósitos e Fundamentos dos coletivos do Hip-Hop

4.3 Identidades dos líderes dos movimentos culturais

4.4. Hip-Hop: instrumento de luta? Transformação social?

5. Aproximações e Distanciamentos dos Movimentos Culturais de Hip-Hop

6. Referências Bibliográficas 
Tain't what you do, it is the way that you do it! Melvin "Sy" Oliver e James Trummer Young It don't mean a thing if it ain't got that swing! Duke Ellington

A one, a two, you know what to do! Frankie Manning 